\title{
FLEXIBLE SHEAR STRESS SENSOR SKIN FOR AERODYNAMICS APPLICATIONS
}

\author{
Fukang Jiang, Yong Xu, Tianxiang Weng, Zhigang Han and Yu-Chong Tai \\ Adam Huang*, Chih-Ming Ho* and Scott Newbern** \\ California Institute of Technology, Pasadena, CA 91125, USA \\ *MAE, University of California, Los Angeles, CA 90024, USA \\ **AeroVironment Inc., Simi Valley, CA 93064, USA
}

\begin{abstract}
Packaging for a large distributed sensing system is a challenging topic. Using flexible skin technology solves many of these problems. Combining with the newly developed backside contact technique, sensor packaging is made even easier by completely avoiding the fragile bonding wires. This paper describes the improved flexible MEMS technology and its application to the fabrication and packaging of practical shear stress sensor skins. An airflow separation detection system including these skins, MOSIS bias circuits and a data acquisition unit has been successfully tested in windtunnel and is being used for the aerodynamic study of a MEMS controlled super-maneuverable low-altitude unmanned aerial vehicle (UAV).
\end{abstract}

\section{INTRODUCTION}

Direct measurement of aerodynamic information on the surface of an aerial vehicle is important but difficult. This difficulty is mainly caused by the unavailability of suitable sensors and the related packaging issues. MEMS sensors have great potential for this application because of their small sizes. However, packaging remains a big challenge as the surfaces of an aerial vehicle are never flat. We have previously reported a possible solution of using flexible sensor skin concept that arrays of MEMS sensors can be made into a flexible skin so they can be applied to a 3-D surface like a Scotch tape [1]. However, the packaging of skins itself is a big hurdle for its wide application. The traditional fine wire bonding and electrical wire soldering are still the major means of connecting sensors out. This can be painstaking and messy as well as unreliable as shown in Fig. 1.

Continuing our previous research, we have developed a new skin technology with many much-improved features. For example, the new skin technology uses DRIE to improve yield and allows backside solder bonding to minimize front side roughness. Most importantly, the new skins can be array-bonded to flexible Kapton PCBs thus completely avoiding bonding wires. These improvements are demonstrated in the fabrication and packaging of new shear stress sensor skins which are designed for airflow separation detection on the leading edges of a delta-wing airplane. The overall detection system that includes packaged sensor skins, MOSIS bias circuits and a dataacquisition/signal-processing unit have been developed and successfully tested in windtunnel and in real flight.

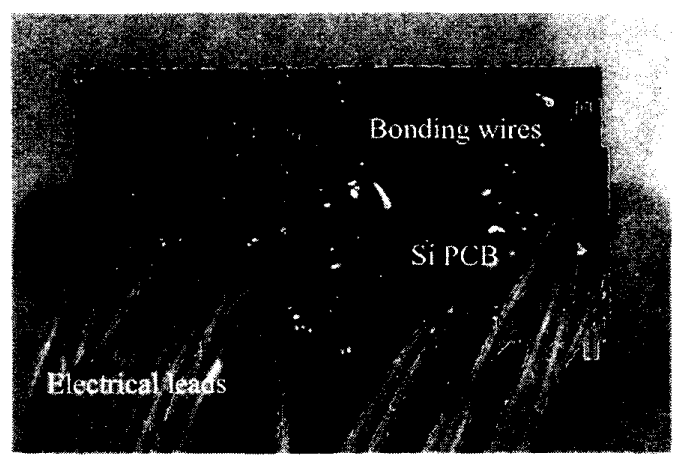

Figure 1 The traditional skin packaging by fine wire bonding and electrical lead soldering.

\section{DESIGN, FABRICATION AND PACKAGING}

The new shear stress sensor skins are designed for flow separation detection on the leading edges of a deltawing airplane (Fig. 12) through shear stress distribution measurement. For a semi-cylindrical leading edge half inch $(12.7 \mathrm{~mm})$ in diameter, the skin should have 36 shear stress sensors spanning the distance of $19.6 \mathrm{~mm}$ to cover the $180^{\circ}$ surface with $5^{\circ}$ resolution. Fig. 2 shows the entire fabrication process of such shear stress sensor skins. DRIE is used to pattern polysilicon sensing wires to improve sensor uniformity due to its very small undercut. In forming Si diaphragms ( $70 \mu \mathrm{m}$ thick), the wet anisotropic etching is replaced by DRIE. Because it does not require front side protection by LPCVD silicon nitride or LTO, this $\mathrm{Si}$ diaphragm formation step can be moved to almost the end of the process so that the wafers or diaphragms have much less chance to break. DRIE is also used in forming $\mathrm{Si}$ islands. Because of its great etching selectivity of silicon over silicon nitride, no aluminum etch stop is necessary. Not only the process is greatly simplified, it also improves the reliability because the remaining 
aluminum etch stop underneath electrical leads separated by an insulation layer could potentially cause short circuit if there are defects on the insulation layer.

1. Deposit and pattern nitride. Local oxidation.

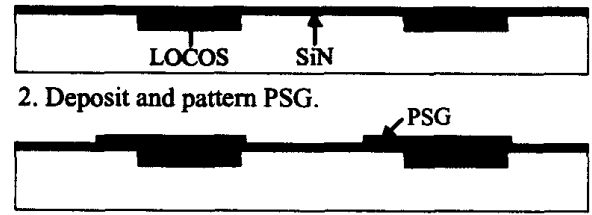

3. Deposit thick nitride and open etch holes;

High concentrated HF removes oxide and PSG.

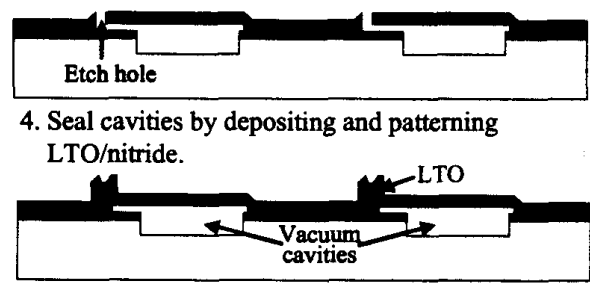

5. Deposit, dope and pattern polysilicon.

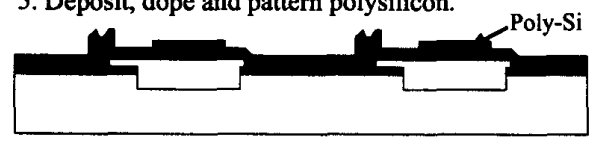

6. Deposit thin nitride and open contact holes; Metallization.

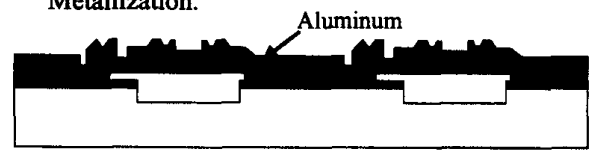

7. Spin on, cure and pattern polyimide at $350^{\circ} \mathrm{C}$.

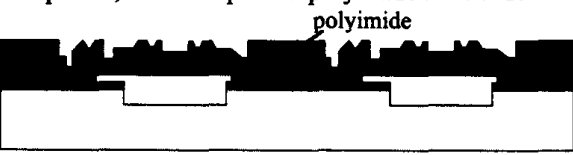

8. Pattern backside; RIE etches nitride; DRIE etches Si to $70 \mu \mathrm{m}$ thick.

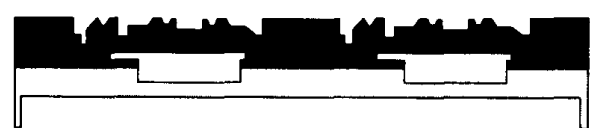

9. DRIE etches away silicon between islands; RIE removes nitride.

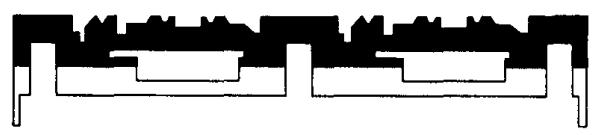

10. Spin, pattern and cure polyimide on the backside. Electroless plate nickel/gold on backside pads.

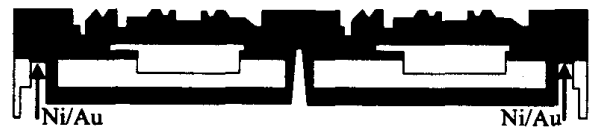

Figure Fabrication process flow.
All the sensor leads are connected to the large aluminum pads at the flexible ends of a skin. There are two rows of pads on each end so that the pad sizes can be made large enough within a very limited area for successful solder bonding. After polyimide is coated on the backside of the skins at step \#10, one can choose to open the contacts on the front side or on the backside. Here we have chosen the latter because it allows the non-flip chip bonding between a skin and PCBs (rigid or flexible). We know that flip-chip bonding is getting popular for IC chip packaging due to its high efficiency and reliability. IC chips can be flip-bonded because the front surface does not have to be exposed to environment. Only front gold pads are needed in that case. However, a lot of MEMS devices such as flow sensors and pressure sensors must have their front surfaces exposed to the environment. The solution is then the non-flip chip bonding in which the backside contacts are solder-bonded to PCBs. In this particular case, non-flip chip bonding has an additional advantage of reducing front surface roughness which is very important for shear stress measurement.

When opening backside contacts, we can not use oxygen plasma to etch polyimide unless a good spray photoresist coating system is available because spincoated photoresist on the very rough backside is extremely non-uniform. Fortunately we can wet etch the uncured polyimide (Du Pont PYRALIN ${ }^{\circledR}$ PI2808) with less accurate definition of patterns which is not a problem since the pads are on the order of a few hundred microns. The photoresist is then removed by butyl acetate, a solvent more gentle than acetone, and alcohol. After polyimide is cured and front side is protected by photoresist, additional steps are used to electrolessly plate nickel/gold on the exposed aluminum pads on the backside. The for electrolessly plating nickel/gold on top of aluminum were developed by Stapleton Technologies Inc. [4] and are widely used for making solder bump on ICs for flip-chip bonding. The recipes and chemicals are available from Technic Inc.. The finished sensor skins are then cut off from wafers by either laser cutting (very precise) or by razor blade (for large size only).

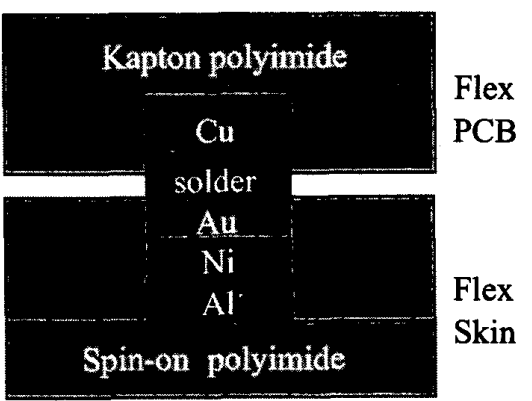

Figure 3 Solder bonding between flexible skins with backside contacts and flexible PCB. 


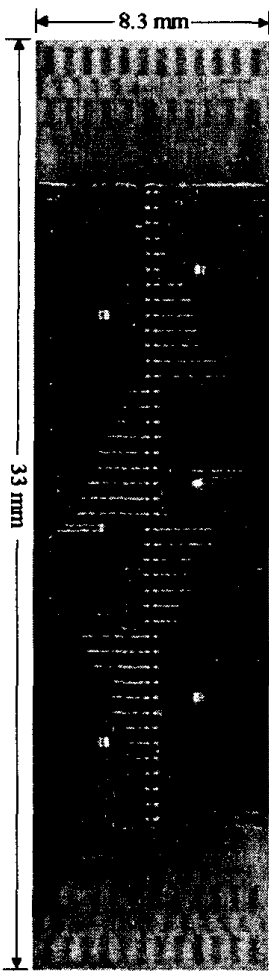

(a)

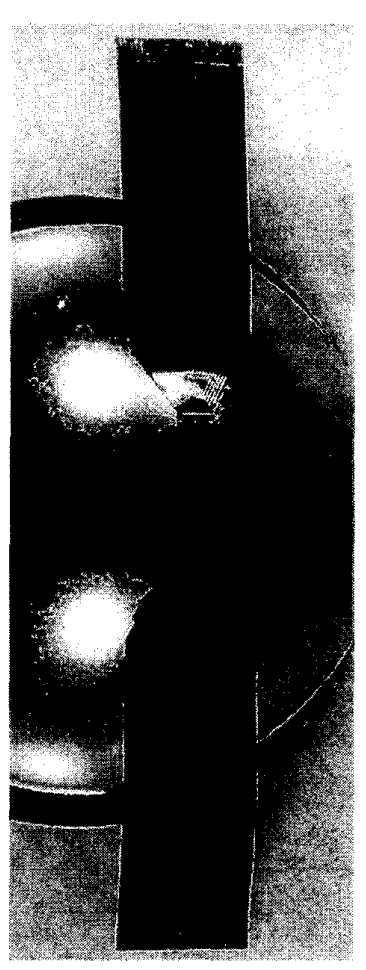

(b)
Figure 4 (a) A new flexible skin with 36 shear stress sensors and backside solder-bonding pads. (b) A new shear stress sensor skin bonded to flexible PCB.

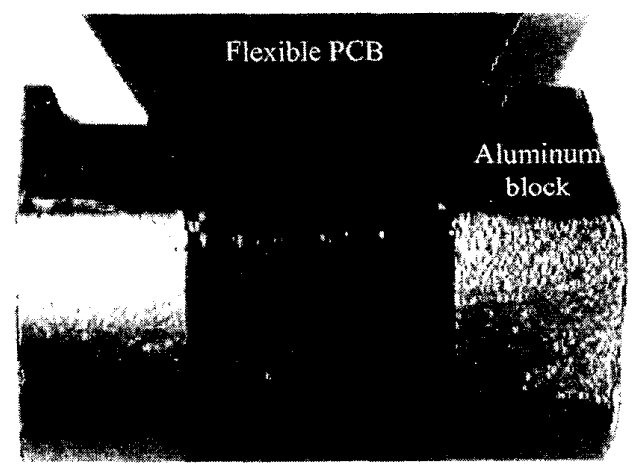

Figure 5 A sensor skin is packaged on a semicylindrical aluminum piece to be mounted on a UAV for flight test.

To demonstrate the non-flip chip bonding idea, we have packaged a skin onto a specially designed flexible PCB, which is $20 \mathrm{~cm}$ long and $3 \mathrm{~cm}$ wide with small solderbonding pads on the narrow part in the middle and large soldering pads by the sides for connector attachment. Fig. 4 shows the pictures of a skin before and after it is bonded to a flexible PCB. The bonding process is simple. First apply solder to the pads and thin RTV to the non-contact area on the backside of the skin and firmly attach it to the flexible PCB with corresponding soldering pads aligned. After the RTV is cured, lay the PCB flat on top of a hotplate and apply uniform force to the pad areas through glass slides. Heat up the hotplate to $200^{\circ} \mathrm{C}$ and then cool down. Fig. 5 shows the bonded skin and flexible PCB mounted on a semi-cylindrical aluminum piece that is cut from the leading edge of a delta wing.

\section{BIAS CIRCUITS}

Hot film sensors can be biased in constant current and constant temperature modes. Constant temperature bias is more often used due to its higher frequency response and sensitivity which are crucial to many fluid mechanics measurements even though the bias circuit is much more complicated than the simple constant current circuit. It also requires accurate adjustment of operating point and hence is more sensitive to ambient temperature change and sensor resistance drift. For these reasons, constant temperature mode should be avoided if high frequency response is not necessary, especially when a large quantity of sensors are involved, such as in this case. We found that in determining a flow separation point, a low frequency sensor can do the job equally well as a high frequency sensor. Therefore, we choose the simple constant current bias mode for this application to greatly reduce the system complexity. In fact, since the sensors can be made with good uniformity, we are able to avoid individual adjustment of sensor operating points by using the multi-channel constant current mirror fabricated using a $2 \mu \mathrm{m}$ CMOS process through MOSIS service. Figs. 6 and 7 are the circuit diagram and the chip photomicrograph $(2 \times 2$ $\mathrm{mm}^{2}$ ). The cascoded transistor pair is for high output impedance and the large transistor area is for minimizing $V_{G S}$ as well as for good uniformity. The output current in each channel is $I_{r e f} / 2$, where $I_{r e f}$ is the reference current and is set by an external three-terminal adjustable voltage regulator $\mathrm{LM} 317 \mathrm{~L}$ and a resistor $R_{r}$. Approximately,

$$
I_{\text {ref }}=\frac{1.25 \mathrm{~V}}{R_{r}}
$$

Table 1 lists the major specifications of this MOSIS current mirror chip.

Table 1 specifications of the current mirror

\begin{tabular}{|c|c|}
\hline $\begin{array}{c}\text { Maximum power } \\
\text { supply voltage }\end{array}$ & $10 \mathrm{~V}$ \\
\hline Current uniformity & $\pm 1 \%$ \\
\hline$V_{G S}(I=2 \mathrm{~mA})$ & $3 \mathrm{~V}$ \\
\hline $\begin{array}{c}\text { Output impedance } \\
(I=2 \mathrm{~mA})\end{array}$ & $300 \mathrm{k} \Omega$ \\
\hline Number of channels & 36 \\
\hline
\end{tabular}




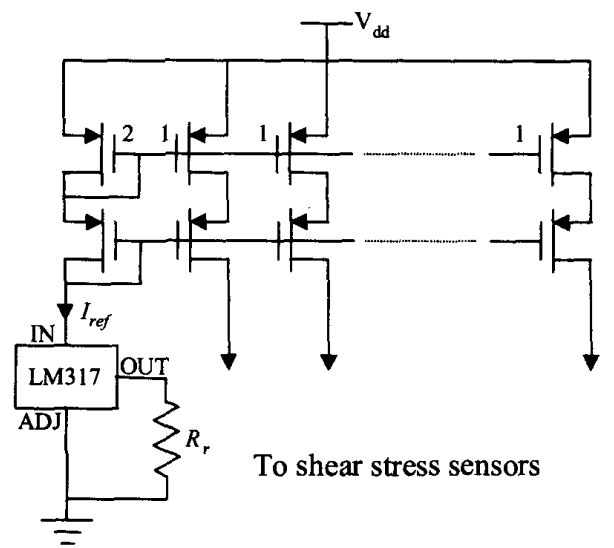

Figure 6 Constant current bias circuits consisting of a MOSIS current mirror and a three terminal adjustable voltage regulator LM317.

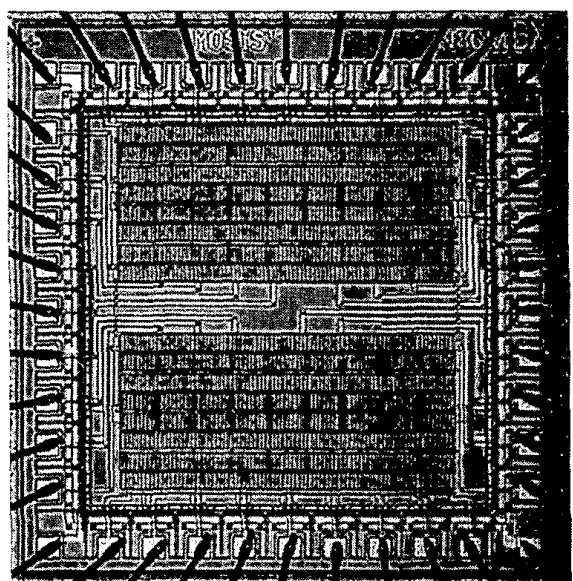

Figure 7 Picture of a MOSIS constant current bias chip.

\section{DATA ACOUISITION SYSTEM}

Fig. 8 is the block diagram of the data acquisition, signal processing and control system. Up to 200 sensor outputs can be multiplexed into a 14-bit A/D converter through a high input impedance JFET buffer stage. Each sensor is sampled at $1 \mathrm{kHz}$ and every 100 digitized data points from each sensor are then processed by a DSP chip (TI C50) to generate the mean and RMS (or standard deviation) value at a rate of $10 \mathrm{~Hz}$. The RMS values for all the sensors in one skin are then compared to each other to locate the separation point. This information is used to control the micro balloon actuators [2]. The complete system including the sensors and the bias board (Fig. 9) has an overall noise level of $0.25 \mathrm{mV}$ rms. Note that the power supplies for the bias board and the data acquisition board must be isolated from each other in order to reduce the analog and digital circuit interference noise.

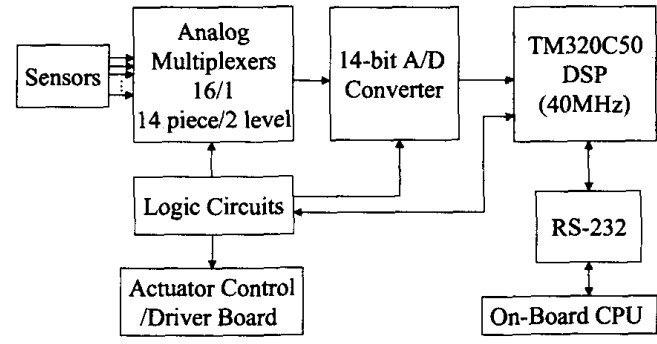

Figure 8 Block diagram of the UAV sensing, data acquisition, signal processing and control system.

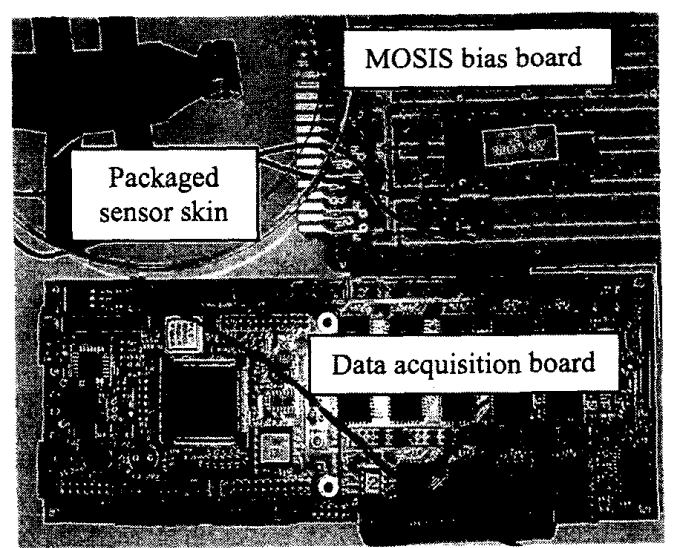

Figure 9 Picture of the complete system, including a sensor skin packaged on a delta wing leading edge section, a MOSIS bias circuit and the 200-channel data acquisition board.

\section{WINDTUNNEL TESTS}

A selected sensor skin has been calibrated in a 2-D windtunnel [3] when flush-mounted on the wall. Figure 10 is the calibration result of a sensor biased in constant current mode at different over-heat ratios. To obtain high signal to noise ratio while still keeping the drift to low level, we decide to fix the over-heat ratio at $20 \%$ in all the subsequent applications.

A sensor skin mounted on a leading edge section has also been tested on a delta-wing airplane model in a wind-tunnel [1]. The DC voltage changes and the RMS voltages of all sensors are plotted in Fig. 11 for an airflow speed of $10 \mathrm{~m} / \mathrm{s}$ and angle of attack (AOA) of $25^{\circ}$. It is known that after flow separates from leading edge surface, the mean shear stress drops to small value while its fluctuation increases dramatically. Therefore either DC or RMS outputs of the sensor array can be used to determine the separation point. However, in order to eliminate the random error, we combine the DC and RMS data to generate the separation point. The separation point is now defined as the point where the DC voltage change starts to drop sharply while the RMS 
voltage is rising to its first peak. In this case, it is at $95^{\circ}$. Note that this is not a strict physical definition. It is only for the study of separation point movement along the delta wing leading edge and with flight speed and AOA.

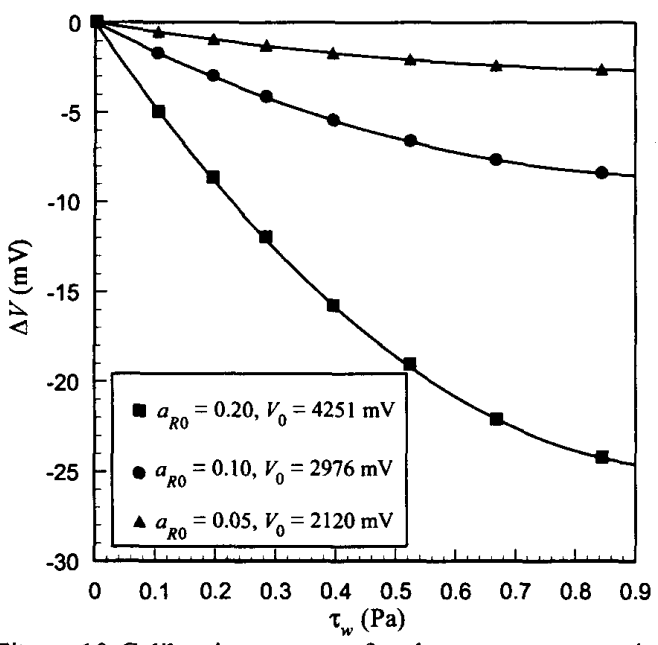

Figure 10 Calibration curves of a shear stress sensor in constant current mode, where $a_{R 0}$ is the over-heat ratio at no flow condition.

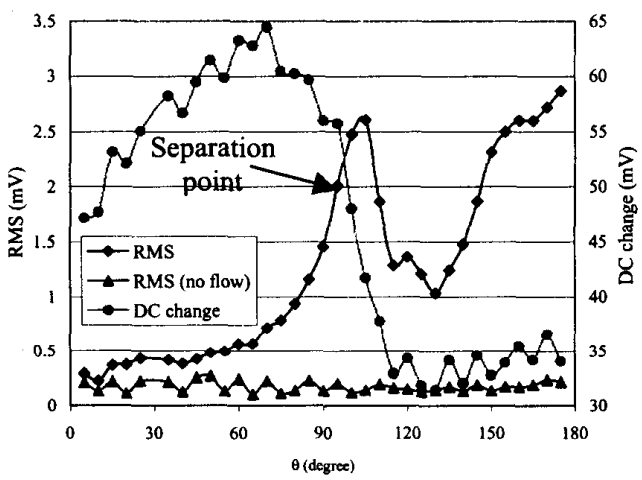

Figure 11 Flow separation detected by a sensor skin biased in constant current mode at an over-heat ratio of $20 \%$ in windtunnel. The airflow speed is $10 \mathrm{~m} / \mathrm{s}$ and the angle of attack is $25^{\circ} . \theta$ is $0^{\circ}$ at the bottom of the wings and $180^{\circ}$ on the top of the wings.

We have also developed a computer algorithm to automatically identify the separation point. The idea is to first find the laminar flow region where the RMS values are all low by comparing all sensor outputs on one skin. The next step is to locate the significant rising section and the peak immediately next to the laminar flow region. The point in this rising section that has half of the peak RMS value is the separation point. This algorithm has been verified by large amount of windtunnel data and will eventually implemented in on- board software for real time MEMS flight control.

\section{FLIGHT TESTS}

As the first step, one sensor skin with 34 working sensors along with the bias circuits and data acquisition board has been mounted on a real delta-wing UAV (Fig. 12) for flight test. The aircraft has a wing span of 71.6 in, a root chord of $53.3 \mathrm{in}$, and weighs $22 \mathrm{lbs}$. It is powered by an internal combustion engine that drives a ducted fan and is remotely piloted. The flight computer and sensor suite includes a full set of transducers that measure aircraft motion and performance parameters. The data is sampled at $100 \mathrm{~Hz}$ and is recorded on-board during the flight. Data is also downlinked in real-time to a ground station for monitoring.

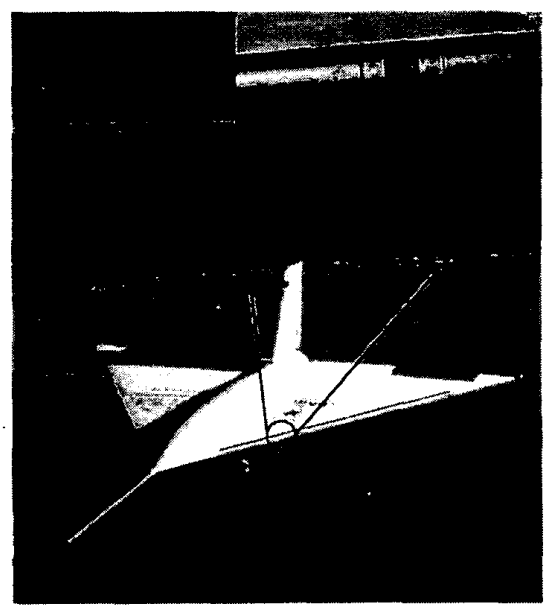

Figure 12 Picture of the UAV.

The amount of data taken during a flight is huge and we can only show a fraction of them here. Fig. 13 shows the flight speed and angle of attack data together with the outputs from sensors \#5 and \#34 which are located near the bottom and the upper surfaces of the wing respectively. It is obvious that sensor \#5 is in the laminar flow region and \#34 is in turbulent flow (vortex) region at most flight conditions, as we have expected. The spikes on sensor \#5 RMS trace is caused by the roll of the aircraft which is reflected on the AOA oscillations around $237 \mathrm{~s}$ and $335 \mathrm{~s}$. Fig. 14 then shows the sensor array outputs at certain moments when the flight speed and AOA are in steady state. We can see that the separation points moves from upper surface of the wing (with large $\theta$ ) towards the bottom surface of the wing (small $\theta$ ) when AOA increases. We also notice that the RMS values from the few sensors in the very beginning (small $\theta$ ) are unexpectedly large. This may be due to the non-ideal factors such the cross flow travelling along the leading edges and the slight yawing of the aircraft during flight. More flight tests are needed to find out the exact causes. 


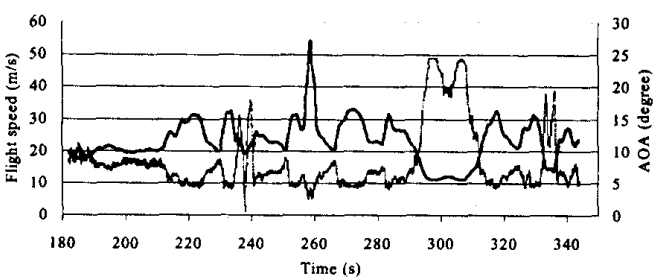

(a) Flight data (speed and AOA)

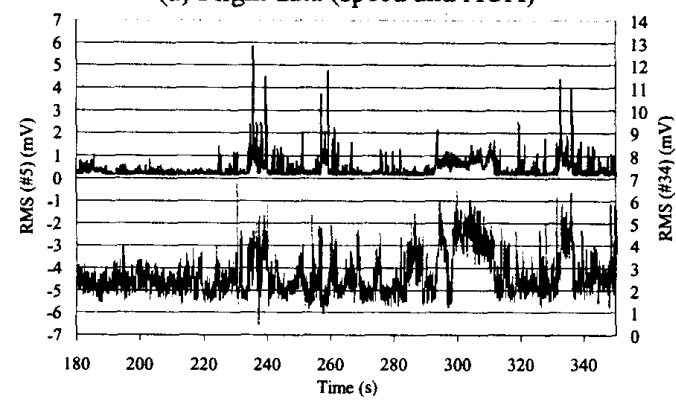

(b) Selected shear stress sensor data.

Figure 13 Flight data and RMS data of selected sensors (\#5 and \#34) from one flight (from take-off to landing).

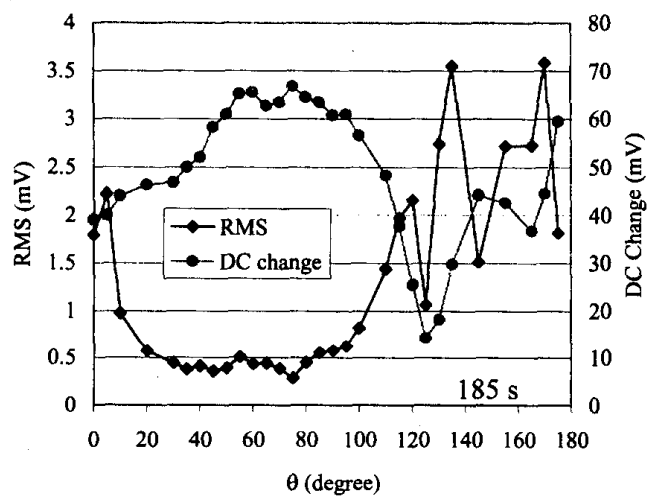

(a) AOA: $10^{\circ}$; speed: $17.5 \mathrm{~m} / \mathrm{s}$. separation point: $95^{\circ}$.

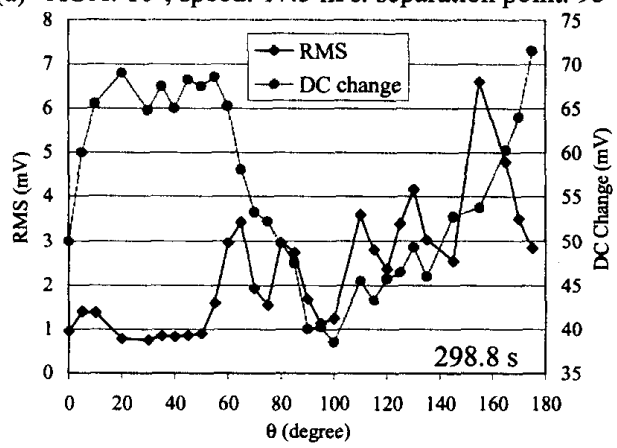

(b) AOA: $24^{\circ}$.; peed: $11 \mathrm{~m} / \mathrm{s}$; s separation point: $55^{\circ}$.

Figure 14 Flow separation point change with AOA. Speed has been found to have little effect on the change of separation point location.

\section{CONCLUSION}

We have developed an improved flexible MEMS technology for making practical shear stress sensor skins and solved the packaging problem for these skins with large amount of sensors using backside contact and non-flip chip solder bonding techniques. An airflow separation detection system including sensor skins, bias circuits and data acquisition unit has been successfully tested in windtunnel and is being used in aerodynamic study of an experimental UAV. This system will eventually be for the real-time maneuvering control of the aircraft.

\section{ACKNOWLEDGMENTS}

This work is supported by DARPA under Navy Contract N66001-97-C-8610. The authors would like to thank Trevor Roper and Hung Bui for fabrication assistance, Chen-Wei Chiu from Umachines Inc. for electroless $\mathrm{Ni} / \mathrm{Au}$ plating, Dave Ganzer, Emil Ghapgharan and Mark Levoe for the UAV flight test.

\section{REFERENCES}

[1] F. Jiang, K. Walsh, T. Tsao, Y.C. Tai, G.B. Lee and C.H. Ho, "A Flexible MEMS Technology and Its First Application to Shear Stress Sensor Skins", 10th International IEEE Workshop on MEMS (MEMS'97), Nagoya Castle, Japan, pp. 465-470, 1997.

[2] C. Grosjean, G.B. Lee, W. Hong, Y.C. Tai and C.M. Ho, "Micro Balloon Actuators For Aerodynamic Control", 11th International IEEE Workshop on MEMS (MEMS'98), Heidelberg, Germany, January 25-29, pp. 166-171, 1998.

[3] F. Jiang, Y.C. Tai, B. Gupta, R. Goodman, S. Tung, J.B. Huang and C.H. Ho, "A Micromachined Shear Stress Sensor Array," 9th International IEEE Workshop on MEMS (MEMS'96), San Diego, USA, pp. 110-115, 1996

[4] MICRO-TECH: Flip Chip Under Bump Metallization, Stapleton Technologies Inc. Technical Notes, 1997. 